\title{
Expression of V-set immunoregulatory receptor in malignant mesothelioma
}

\author{
Yeon Seung Chung ${ }^{1} \cdot$ Moonsik Kim $\mathbb{B}^{1} \cdot$ Yoon Jin Cha $\mathbb{D}^{1} \cdot \operatorname{Kyung~A~Kim}^{1} \cdot$ Hyo Sup Shim $\mathbb{D}^{1}$
}

Received: 15 April 2019 / Revised: 20 June 2019 / Accepted: 21 June 2019 / Published online: 30 July 2019

(c) United States \& Canadian Academy of Pathology 2019

\begin{abstract}
Malignant mesothelioma is a highly lethal cancer. V-set immunoregulatory receptor (VSIR, also known as V-domain Ig suppressor T-cell activation, VISTA), a negative immune checkpoint regulator, was reported to be expressed in malignant mesothelioma; however, its detailed expression pattern and clinicopathological significance have not been elucidated. We examined the expression of VSIR and CD274 and CD8 ${ }^{+}$tumor-infiltrating lymphocytes in a total of 124 samples from 66 patients with malignant mesothelioma and analyzed the clinicopathological characteristics and their relationship with the immunohistochemical findings. A total of 553 non-small cell lung carcinomas were also evaluated for VSIR expression. VSIR expression was higher in epithelioid type mesothelioma $(p<0.001)$, whereas CD274 expression was higher in sarcomatoid type $(p<0.001)$. CD8 ${ }^{+}$tumor-infiltrating lymphocytes were more abundant in sarcomatoid mesotheliomas $(p<0.001)$, VSIR-low tumors $(p=0.045)$, and CD274-high tumors $(p<0.001)$. VSIR and CD274 were differentially expressed in each histological component of the biphasic type. VSIR expression was associated with favorable survival $(p=0.008)$. Two patients with VSIR-high tumors had received pembrolizumab; however, they showed progressive disease. No VSIR expression was observed in tumor cells of non-small cell lung carcinomas. In conclusion, VSIR expression may define a unique class of mesothelioma, characterized by predominantly epithelioid type and favorable prognosis. VSIR expression may be used as an immunohistochemical diagnostic marker for epithelioid mesothelioma. CD274 expression was associated with sarcomatoid mesothelioma and high infiltration of $\mathrm{CD} 8^{+}$lymphocytes. Because VSIR is a negative immune regulator and expressed in malignant mesothelioma, further study is warranted to investigate the therapeutic significance of VSIR blockade in this deadly cancer.
\end{abstract}

\section{Introduction}

Malignant mesothelioma is a rare and highly lethal cancer [1]. The 5-year survival rate of patients with unresectable mesothelioma is reported to be $<5 \%$ [2]. To date, the only approved frontline regimen for unresectable tumors is cisplatin-pemetrexed [1]. Recently, the results of clinical trials for treatment with immune checkpoint inhibitors have been reported, and this new treatment option is attracting

Supplementary information The online version of this article (https:// doi.org/10.1038/s41379-019-0328-3) contains supplementary material, which is available to authorized users.

Hyo Sup Shim

shimhs@yuhs.ac

1 Department of Pathology, Yonsei University College of Medicine, Seoul, Korea attention [3, 4]. In addition, in-depth research into the genomic alterations of mesotheliomas to understand mesothelioma biology and identify novel therapeutic options is underway [5-7].

A recent integrated genomic study showed that V-set immunoregulatory receptor (VSIR, also known as V-domain Ig suppressor T-cell activation, VISTA), a negative immune checkpoint molecule, was highly expressed in mesothelioma compared with that in other carcinomas [6]. VSIR is a member of the B7 family of negative checkpoint regulators $[8,9]$. It is expressed on the surface of several immune cells and antigen-presenting cells [8,9] and is known to inhibit early-stage T-cell activation [10]. However, whether VSIR is expressed in cells other than immune cells is not well understood. Furthermore, the relationship between VSIR expression and other immunerelated markers, such as CD274 (programmed cell deathligand 1, PD-L1) expression and $\mathrm{CD}^{+}$tumor-infiltrating $\mathrm{T}$ cells, has not been determined in clinical samples. 
Because differential diagnosis between mesothelioma and non-small cell lung carcinoma is sometimes difficult, and additional diagnostic markers are necessary, VSIR expression in non-small cell lung carcinomas warrants further investigation.

In this study, we examined the expression of VSIR in mesothelioma, as well as non-small cell lung carcinoma using clinical specimens and investigated the clinical and diagnostic significance thereof.

\section{Materials and methods}

\section{Patients}

Medical records and archival slides from a collection of malignant mesotheliomas from patients presenting at our institution between 2001 and 2017 were retrospectively analyzed. The institutional review board approved this retrospective study. Cases were selected from consecutive series on the basis of availability of archival slides and tissue. Clinical data, including age, sex, treatment, and survival, were obtained from each patient's medical records. Stage of pleural mesothelioma was classified according to the 8th edition of the standards of American Joint Committee on Cancer. Stage of peritoneal mesothelioma was classified according to a staging system proposed by Yan et al. [11].

\section{Histological review}

Samples were classified as epithelioid, sarcomatoid, and biphasic mesothelioma, according to the 2015 World Health Organization classification [12].

\section{Immunohistochemistry and interpretation}

Whole sections were used for immunohistochemistry, regardless of the sampling method. Formalin-fixed paraffinembedded tissues were sectioned at a thickness of $4 \mu \mathrm{m}$ and stained with anti-CD274 (anti-PD-L1 mouse monoclonal, clone $22 \mathrm{C}$, dilution 1:50, Agilent, Santa Clara, CA, USA) and anti-VSIR (anti-VISTA rabbit monoclonal, clone D1L2G, dilution 1:100, Cell Signaling Technology, Danvers, MA, USA) antibodies using the Ventana BenchMark XT automated staining platform (Ventana Medical Systems, Tucson, AZ, USA). For CD8 immunohistochemistry, FLEX ready-to-use monoclonal mouse anti-human $\mathrm{CD} 8$ (clone C8/144B, Agilent) was used. Signals were detected using the ultraView Universal DAB Detection Kit (Ventana Medical Systems).

The CD274 and VSIR expression in tumor cells was expressed as the tumor proportion score. Tumor proportion score was defined as the percentage of viable tumor cells showing positive staining relative to all viable tumor cells present in the sample. Only membranous staining was considered positive for CD274 expression. Cytoplasmic and/or membranous staining was considered positive for VSIR expression. Staining with any intensity was considered positive for both markers. $\mathrm{CD}^{+}$tumorinfiltrating lymphocytes were defined as cells with immunoreactivity for CD8 in both the stromal and intratumoral compartment of the tumor. The number of tumorinfiltrating lymphocytes per high-power field was determined manually in up to four independent tumor areas showing the highest lymphocyte density. The average cell counts per high-power field were recorded for each slide. Cytology samples were excluded from $\mathrm{CD}^{+}$tumorinfiltrating lymphocyte evaluation. Immunohistochemistry interpretation was performed by two pathologists (YSC and HSS). Discrepancies in interpretation were resolved by consensus discussion. All 124 samples from 66 patients were separately evaluated for histology, VSIR, $\mathrm{CD} 274$, and $\mathrm{CD}^{+}$tumor-infiltrating lymphocytes. Each score was used for comparison with histology or other factors, and average scores were used for survival analysis of patients.

\section{Tissue microarrays of non-small cell lung carcinomas}

After histological review of non-small cell lung carcinomas, two cores of 3-mm diameter were taken from formalin-fixed paraffin-embedded tissue blocks, and tissue microarrays were constructed for immunohistochemistry. Two cores were sampled from histologically different areas; one from the center of tumor and the other from the edge.

\section{Statistical analysis}

Relationships between clinicopathological parameters were evaluated using the Chi-squared test. Comparison between two groups with continuous values was performed using an unpaired t-test. Intra-class correlation coefficient estimates and their $95 \%$ confident interval were calculated by reliability analysis using IBM SPSS Statistics v.23 (IBM, Armonk, NY, USA). When multiple samples were available for a patient, the patient was classified according to the average scores of VSIR, CD274, and $\mathrm{CD}^{+}$tumorinfiltrating lymphocytes, and the association with survival was examined. Overall survival was evaluated using the Kaplan-Meier method, and statistical differences in survival times were determined using the log-rank test. Coxproportional hazards model was applied for multivariate survival analysis. Differences were considered significant at $p<0.05$. All statistical analyses were conducted using IBM SPSS Statistics v.23. 
Table 1 Patient cohort in this study according to histological type

\begin{tabular}{lllll}
\hline & Total & Epithelioid & Sarcomatoid & Biphasic \\
\hline Patients (\%) & $66(100)$ & $50(77)$ & $11(17)$ & $5(8)$ \\
Sex & & & & \\
$\quad$ Male & $44(67)$ & $32(64)$ & $9(82)$ & $3(60)$ \\
Female & $22(33)$ & $18(36)$ & $2(18)$ & $2(40)$ \\
Age & & & & \\
$\quad$ Average & $61( \pm 14)$ & $62( \pm 15)$ & $57( \pm 11)$ & $58( \pm 11)$ \\
$\quad$ Median & 63 & 64 & 60 & 57 \\
Site & & & & \\
Pleura & $50(76)$ & $38(76)$ & $9(82)$ & $3(60)$ \\
$\quad$ Peritoneum & $16(24)$ & $12(24)$ & $2(18)$ & $2(40)$ \\
Stage & & & & $2(40)$ \\
I & $13(20)$ & $9(18)$ & $2(18)$ & $1(20)$ \\
II & $8(12)$ & $7(14)$ & $0(0)$ & $1(20)$ \\
III & $8(12)$ & $5(10)$ & $2(18)$ \\
IV & $37(56)$ & $29(58)$ & $7(64)$ & $1(20)$ \\
Neo-adjuvant chemotherapy & & & \\
$\quad$ Administration & $10(15)$ & $8(16)$ & $0(0)$ & $2(40)$ \\
Samples, No. (\%) & $124(100)$ & $93(75)$ & $19(15)$ & $12(10)$ \\
Biopsy & $78(63)$ & $59(63)$ & $15(79)$ & $4(33)$ \\
Excision & $36(29)$ & $25(27)$ & $4(21)$ & $7(58)$ \\
Cytology & $10(8)$ & $9(10)$ & $0(0)$ & $1(8)$ \\
\hline & & & &
\end{tabular}

\section{Results}

\section{Patient characteristics}

A total of 66 patients were enrolled. Patient characteristics are summarized in Table 1. Malignant mesothelioma samples were derived from 44 men $(67 \%)$ and 22 women $(33 \%)$, aged $19-85$ years (mean $=61$ years). Three patients had history of asbestos exposure. Fifty patients (77\%) had epithelioid type mesothelioma, 11 (17\%) sarcomatoid type, and five (8\%) biphasic type. Fifty patients $(76 \%)$ had pleural mesothelioma, and 16 (24\%) had peritoneal mesothelioma. Thirteen patients (20\%) were classified as stage I, eight (12\%) were stage II, eight (12\%) were stage III, and $37(56 \%)$ were stage IV. Ten patients $(15 \%)$ received preoperative chemotherapy. A total of 124 samples (mean = 2 samples/patient, range of 1-5) were obtained. Seventyeight samples (63\%) were from biopsy, 36 (29\%) were from excision, and $10(8 \%)$ were for cytology. Types of excision are described in Supplementary Data 1.

\section{VSIR expression}

VSIR was expressed in the cytoplasm and/or membrane of both malignant and benign mesothelial cells. In tumor cells, the expression was higher in epithelioid type than in sarcomatoid type $(p<0.001)$ (Figs. 1 and $2 a)$. When $5 \%$ and $50 \%$ were set as positive cutoffs, $94 \%$ and $78 \%$ of epithelioid type samples were positive for VSIR expression, respectively (See Figure, Supplementary Data 2, which shows the positive rate of VSIR and CD274). In contrast, $55 \%$ and $0 \%$ of sarcomatoid type samples were positive, respectively. In biphasic type, VSIR was predominantly expressed in the epithelioid component (see Figure, Supplementary Data 3, containing representative photos of biphasic mesothelioma). There was no significant difference in VSIR expression between pleural mesothelioma and peritoneal mesothelioma $(p=0.175)$ (see Figure, Supplementary Data 4A, which shows VSIR expression in epithelioid type according to tumor site). Among sample types in epithelioid mesothelioma, there were no significant differences in VSIR expression between biopsy and excision $(p=0.123)$ or between biopsy and cytology $(p=0.194)$ (See Figure, Supplementary Data 4B, which shows VSIR expression in epithelioid type according to sample type). However, excision samples showed higher VSIR expression compared with cytology samples $(p=0.016)$ (See Figure, Supplementary Data 4B).

\section{CD274 expression}

CD274 expression was not present in benign mesothelial cells. In tumor cells, the expression was higher in sarcomatoid type than epithelioid type $(p<0.001)$ (Figs. 1 and $2 \mathrm{~b})$. When $5 \%$ and $50 \%$ were set as positive cutoffs, $18 \%$ and $8 \%$ of epithelioid type samples were positive for CD274 expression, respectively (See Figure, Supplementary Data 2). In contrast, 55 and $46 \%$ of sarcomatoid type samples were positive, respectively. In biphasic type, CD274 expression was higher in the sarcomatoid component (See Figure, Supplementary Data 3). Among sample types in sarcomatoid mesothelioma, excision samples showed higher CD274 expression compared with biopsy samples; however, the difference was not statistically significant $(p=0.243)$ (See Figure, Supplementary Data 4C, which shows CD274 expression in sarcomatoid type according to sample type).

\section{Concordance between observers and among multiple samples from same patients}

Concerning interobserver concordance, intra-class correlation coefficient estimate was 0.966 (95\% confident interval: $0.952-0.976, p<0.001)$ for VSIR and 0.958 (95\% confident interval: $0.940-0.971, p<0.001)$ for CD274, respectively. This indicated excellent reliability. In contrast, among multiple samples from same patients, intra-class correlation coefficient estimate was 0.920 (95\% confident interval: $0.840-0.960, p<0.001$, good to excellent reliability) for 


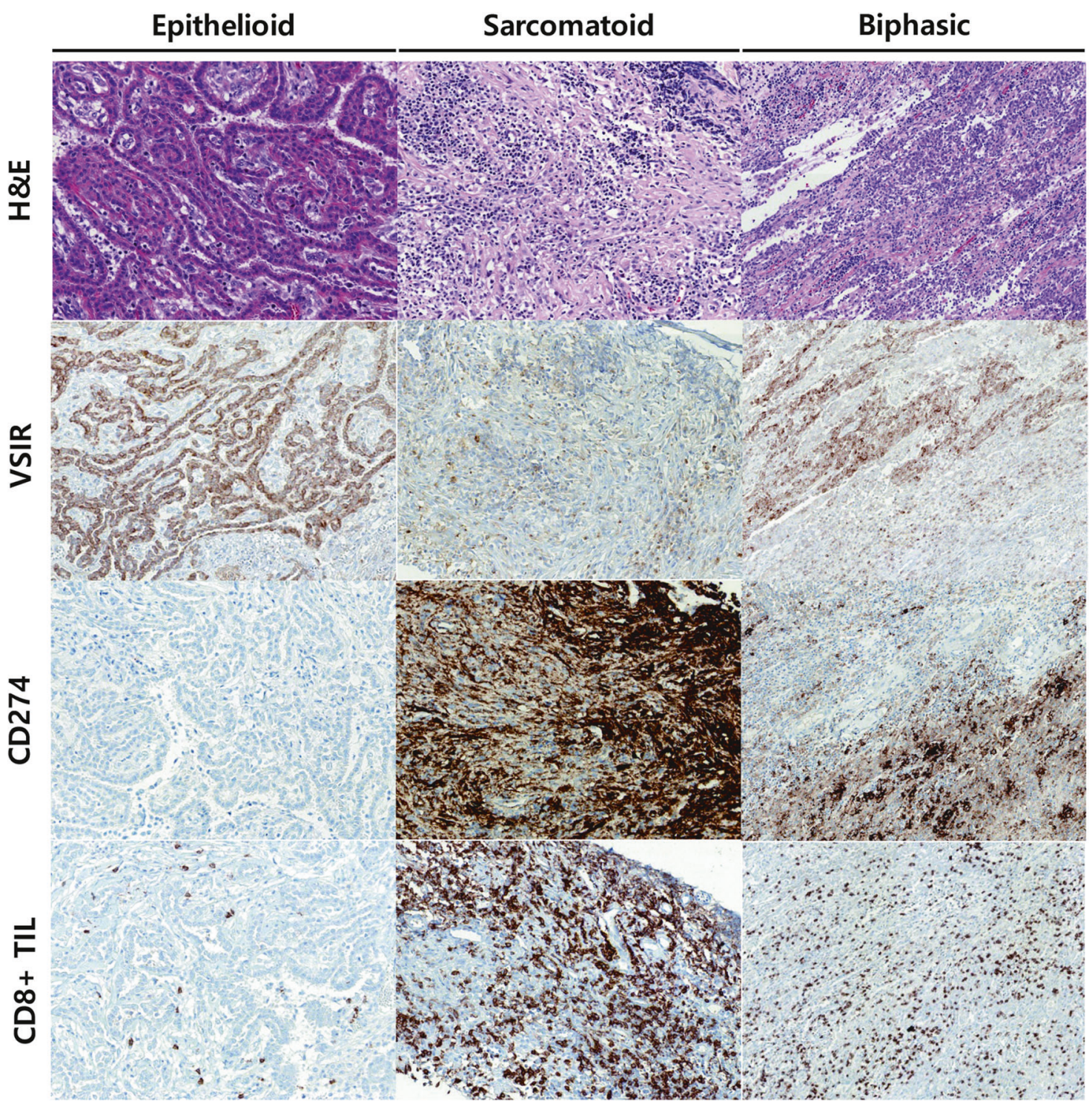

Fig. 1 Representative photos of immunohistochemistry for VSIR, CD274, and CD8 according to histologic type of malignant mesothelioma

VSIR and 0.776 (95\% confident interval: $0.552-0.888, p<$ 0.001 , moderate to good reliability) for CD274.

\section{CD8 + tumor-infiltrating lymphocytes}

$\mathrm{CD}^{+}$tumor-infiltrating lymphocytes were more abundant in sarcomatoid type than in epithelioid type $(p<0.001)$ (Fig. 3a) and in VSIR-low mesotheliomas $(p=0.045)$ and CD274-high mesotheliomas $(p<0.001)$ (Fig. 3b, c).

\section{Prognostic and clinical significance}

The mean follow-up duration was 21 months (range 1-172). There were no significant differences in overall survival according to histological type $(p=0.590), \quad \mathrm{CD} 274$ expression (cutoff: 5\%, $p=0.783$ ), and $\mathrm{CD}^{+}$tumorinfiltrating lymphocytes (cutoff: 152/high-power field, $p=0.522$ ). In contrast, VSIR expression (cutoff: 5\%) was a favorable prognostic factor in a cohort of all types and a subgroup of epithelioid type $(p=0.008$ and $p<0.001$, respectively) (Fig. 4). On multivariate survival analysis including age, sex, stage, subtypes, and sites, low VSIR expression (less than 5\%) was an independent poor prognostic factor ( $p=0.014$, hazard ratio: $3.019,95 \%$ confident interval: 1.25-7.72) (See Table, Supplementary Data 5). Two patients with epithelioid type had received pembrolizumab, a programmed cell death 1 immune checkpoint inhibitor; however, they showed progressive disease. In addition, $70 \%$ and $100 \%$ of their tumor cells showed VSIR expression, respectively, with no CD274 expression. 


\section{VSIR expression in non-small cell lung carcinomas}

VSIR was not definitely expressed in tumor cells in a total of 553 non-small cell lung carcinomas, consisting of 404 adenocarcinomas, 132 squamous cell carcinomas, and 17 large cell carcinomas (Fig. 5). Only two cases showed patchy cytoplasmic staining of less $5 \%$ of tumor cells.

\section{Discussion}

Malignant mesotheliomas are a highly lethal cancer with a poor prognosis in the advanced stage. They are largely characterized by loss of tumor suppressor genes, such as BAP1, NF2, TP53, LATS2, and CDKN2A. Druggable mutations, which are an indication of targeted therapies, are very rare. Recently, the effects of immune checkpoint inhibitors on malignant mesothelioma have been reported

A

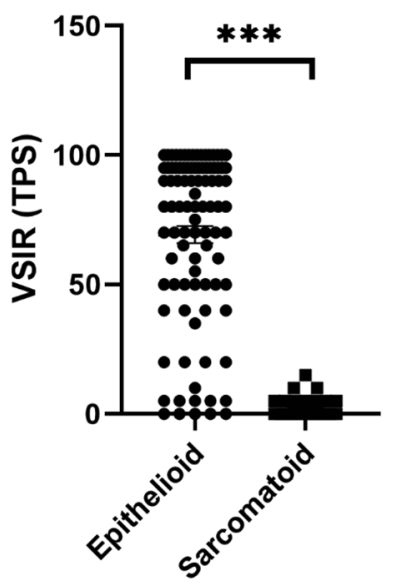

B

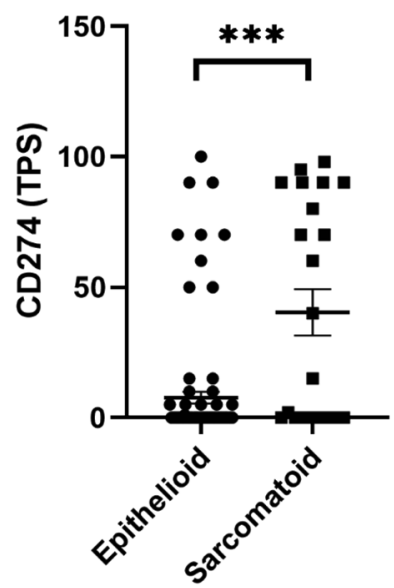

Fig. 2 Scatter dot plot showing tumor proportion scores (TPS) according to histologic type. a VSIR, b CD274; biphasic types were excluded in this comparison; $* * * p<0.001$

Fig. 3 Scatter dot plot showing number of $\mathrm{CD}^{+}$tumorinfiltrating lymphocytes per high-power field (HPF) according to histologic type and expression of VSIR and CD274. a histologic type, b VSIR, cutoff $5 \%$, c CD274, cutoff $5 \%$; cytology specimens were excluded for CD8 scoring; $* * * p<0.001 ; * p=0.045$
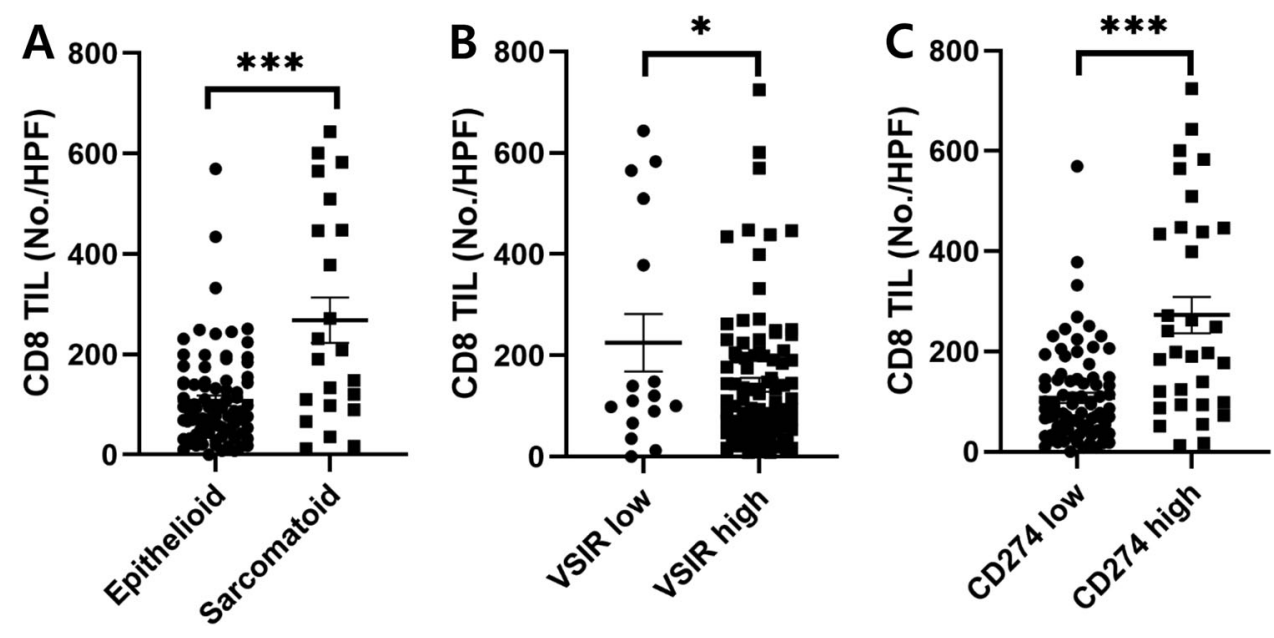

$[3,4,13]$. However, because only $20 \%$ of patients respond to this treatment, predictive biomarkers or a new therapeutic approach is needed [4].

In this study, we confirmed that VSIR is expressed in mesotheliomas, especially in epithelioid type. Epithelioid mesothelioma expressing VSIR infrequently expressed CD274, suggesting that programmed cell death 1/CD274 inhibitors may be less effective in these tumors. In fact, programmed cell death 1 inhibitors were administered to two patients in our cohort diagnosed with epithelioid mesothelioma with high expression of VSIR and no expression of CD274, but no drug effect was observed. Notably, VSIR expression was associated with favorable survival. Preclinical studies of VSIR blockade have shown promising improvement in antitumor T-cell responses [10]. However, further research is needed to determine the effect of VSIR blockade in the treatment of mesothelioma, as well as the role of VSIR in an immunosuppressive environment.

The mechanism of VSIR expression in mesothelioma should also be elucidated. To date, it has been known that VSIR is primarily found in hematopoietic cells, not in epithelial cells [8-10, 14]. Therefore, only a few studies have investigated the role of VSIR protein in tumor cells $[6,15,16]$. According to our observation and other study, VSIR was expressed in benign mesothelial cells as well as in tumor cells of mesothelioma [6]. Thus, the mechanism and regulation of VSIR expression in mesothelium and its malignant tumors should be studied further considering the tissue-dependent microenvironment.

We did not detect definite VSIR expression in tissue microarray sets of non-small cell lung carcinomas. Villarroel-Espindola $\mathrm{F}$ et al. reported VSIR expression in tumor cells in $21 \%$ of non-small cell lung carcinomas [15]. They used an immunofluorescence platform, and described that VSIR expression was predominantly focal in tumor cells. We thought that there might be a number of factors leading to such discrepancy in the results of this study. First,

SPRINGER NATURE 
A

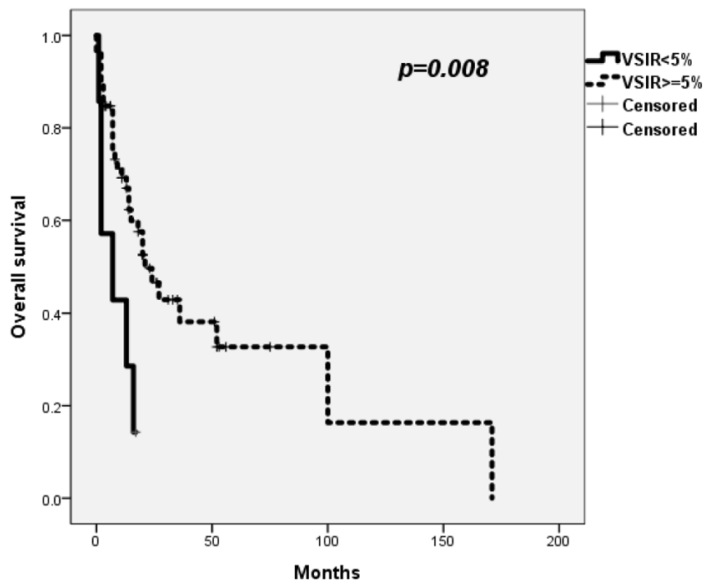

B

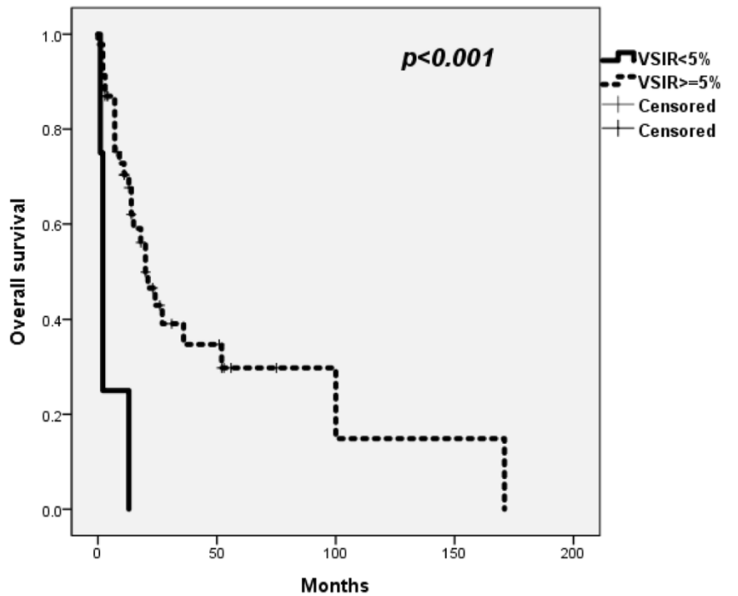

Fig. 4 Overall survival curves according to VSIR expression. a all patients, b patients with epithelioid mesothelioma

there is a possibility that the expression of VSIR could be detected more sensitively by immunofluorescence, due to the difference in the analytic sensitivity between immunohistochemistry and immunofluorescence. Second, the use of tissue microarray in both studies may not perfectly reflect the heterogeneous expression of VSIR in lung cancer. Further studies are needed to determine whether VSIR is expressed in lung cancer cells.

The specific expression of VSIR in epithelioid mesothelioma suggests its promising utility as a marker in diagnostic practice, including in differential diagnosis of mesothelioma and non-small cell lung carcinoma. To confirm the diagnosis of mesothelioma, panels of positive and negative antibodies, including anti-calretinin, D2-40, CK5/ 6 , and WT-1, are used as mesothelioma markers in standard practice. This technique is used because of variability in staining and differential diagnoses [17]. The high sensitivity and specificity of VSIR for epithelioid mesothelioma revealed in this study is expected to facilitate accurate mesothelioma diagnosis.

However, the finding that VSIR is also expressed in benign mesothelial cells suggests that VSIR cannot be used to differentiate between benign mesothelial proliferation and epithelioid mesothelioma. This suggests that VSIR is similar to D2-40, CK5/6, or WT-1 mentioned above in terms of diagnostic markers [17]. In other words, VSIR expression can be regarded as a more differentiated subset of mesothelioma such as the epithelioid type in which the mesothelial lineage is maintained [6]. Until now, special tests have been proposed to differentiate between benign and malignant mesothelial proliferation, including staining for $\mathrm{p} 53$, epithelial membrane antigen, glucose transporter 1, and U3 small nucleolar ribonucleoprotein protein [17]. More recently, homozygous deletion of p16 by fluorescent in situ hybridization or the loss of BRCA1 associated protein 1 by immunohistochemistry has been suggested as a useful adjunct $[18,19]$.

In this study, VSIR was expressed in the cytoplasm and/ or membrane of tumor cells. The reason for the staining with cytoplasmic pattern may be that, according to the antibody manufacturer, monoclonal antibody is produced by immunizing animals with a synthetic peptide corresponding to residues near the carboxy terminus (intracytoplasmic domain) of human VSIR protein.

CD274 expression in tumor cells and the amount of CD8 ${ }^{+}$ tumor-infiltrating lymphocytes were higher in sarcomatoid mesothelioma compared with those in epithelioid type, consistent with the results of previous studies [20, 21]. These results indicate that sarcomatoid mesothelioma may be an immunologically hot tumor and can serve as a suitable target for programmed cell death 1/CD274 immune checkpoint inhibitors. Differential expression of negative immune regulators such as VSIR and CD274 indicates that epithelioid and sarcomatoid mesotheliomas are immunologically distinct. When evaluating the response to programmed cell death 1/CD274 inhibitors, histological subtype should be considered in addition to CD274 status.

In this study, we evaluated concordance of immunohistochemistry scores between observers and among multiple samples from same patients. Interobserver agreement showed excellent reliability. However, concordance among multiple samples, especially for CD274, was relatively low. Taken together, the difference in expression can occur mainly among the samples, especially in CD274, suggesting the heterogeneity of CD274 expression.

This study has several limitations. First, three patients in total had history of asbestos exposure. It was similar to that of a prior report on 66 Korean patients with malignant 


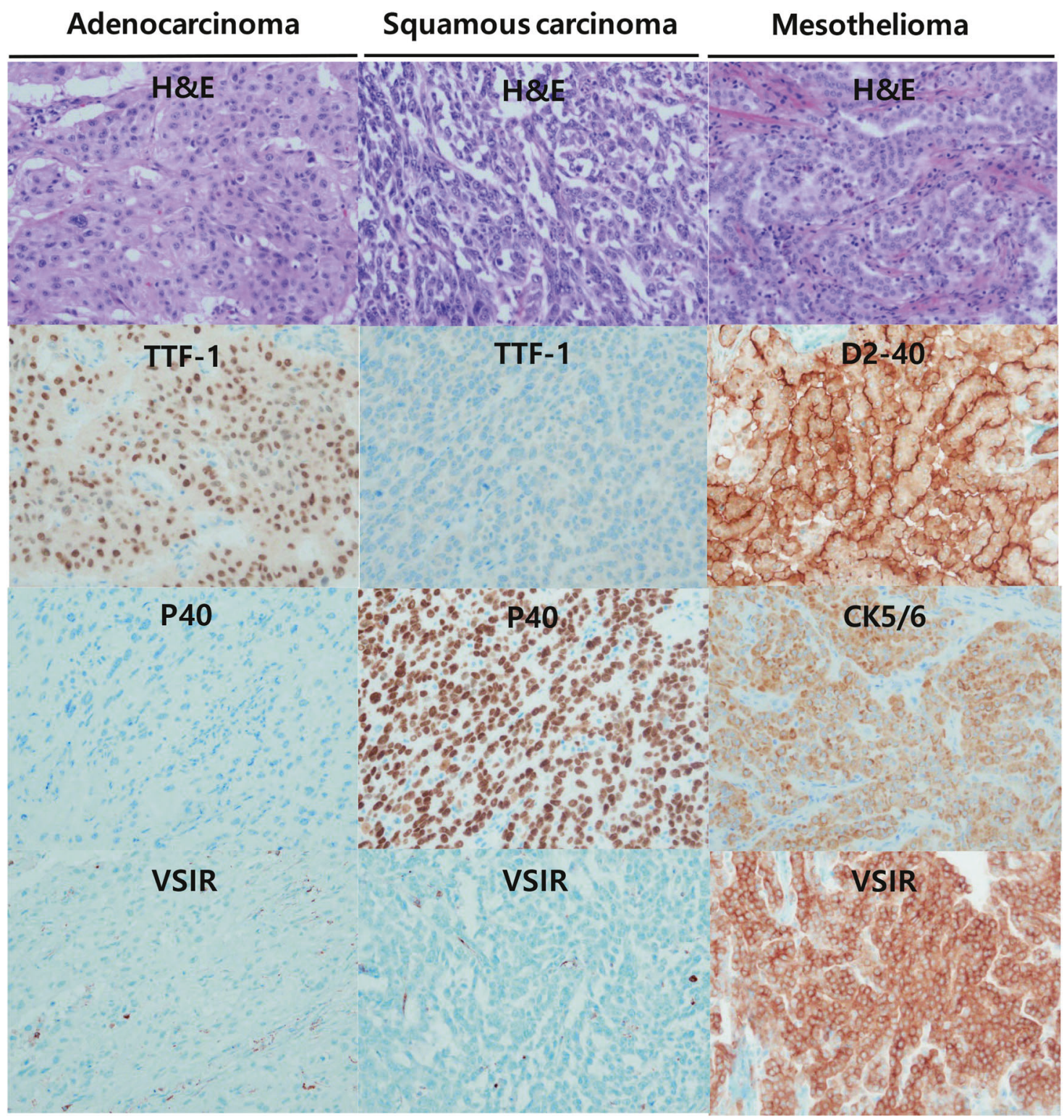

Fig. 5 Representative photos of VSIR expression in cases diagnosed as lung adenocarcinoma ( $1^{\text {st }}$ column), lung squamous cell carcinoma ( $2^{\text {nd }}$ column), and mesothelioma ( $3^{\text {rd }}$ column)

mesothelioma, in which four patients had history of asbestos exposure [22]. Therefore, it was difficult to get statistically significant data of asbestos exposure in our study. Second, the studied cohort was comprised of a small number of patients. Due to the sample size in our cohort, there was no difference in prognosis according to previously known prognostic factors, such as subtypes. Third, only two patients had received programmed cell death 1 inhibitor. Thus, the scope for evaluating the relationship between expression of immune regulators and response to immunotherapy was limited. Further studies are warranted to investigate the significance of VSIR expression in treatment of mesothelioma.
In conclusion, VSIR expression may define a unique class of mesothelioma, characterized by predominantly epithelioid type and favorable prognosis. VSIR expression can be used as an immunohistochemical diagnostic marker for epithelioid mesothelioma. CD274 expression was associated with sarcomatoid mesothelioma and high infiltration of $\mathrm{CD}^{+}$lymphocytes. Because VSIR is a negative immune regulator and expressed in malignant mesothelioma, further study is warranted to investigate the therapeutic significance of VSIR blockade in this deadly cancer.

Acknowledgements This research was supported by Basic Science Research Program through the National Research Foundation of Korea 
(NRF) funded by the Ministry of Education (NRF2018R1D1A1B07047811).

\section{Compliance with ethical standards}

Conflict of interest The authors declare that they have no conflict of interest.

Publisher's note: Springer Nature remains neutral with regard to jurisdictional claims in published maps and institutional affiliations.

\section{References}

1. Tsao AS, Lindwasser OW, Adjei AA, Adusumilli PS, Beyers ML, Blumenthal GM, et al. Current and Future Management of Malignant Mesothelioma: A Consensus Report from the National Cancer Institute Thoracic Malignancy Steering Committee, International Association for the Study of Lung Cancer, and Mesothelioma Applied Research Foundation. J Thorac Oncol. 2018;13:1655-67.

2. Nowak AK, Chansky K, Rice DC, Pass HI, Kindler HL, Shemanski L, et al. The IASLC Mesothelioma Staging Project: Proposals for Revisions of the $\mathrm{T}$ Descriptors in the Forthcoming Eighth Edition of the TNM Classification for Pleural Mesothelioma. J Thorac Oncol. 2016;11:2089-99.

3. Calabro L, Morra A, Giannarelli D, Amato G, D'Incecco A, Covre $\mathrm{A}$, et al. Tremelimumab combined with durvalumab in patients with mesothelioma (NIBIT-MESO-1): an open-label, non-randomised, phase 2 study. Lancet Respir Med. 2018;6:451-60.

4. Alley EW, Lopez J, Santoro A, Morosky A, Saraf S, Piperdi B, et al. Clinical safety and activity of pembrolizumab in patients with malignant pleural mesothelioma (KEYNOTE-028): preliminary results from a non-randomised, open-label, phase $1 \mathrm{~b}$ trial. Lancet Oncol. 2017;18:623-30.

5. Yap TA, Aerts JG, Popat S, Fennell DA. Novel insights into mesothelioma biology and implications for therapy. Nat Rev Cancer. 2017;17:475-88.

6. Hmeljak J, Sanchez-Vega F, Hoadley KA, Shih J, Stewart C, Heiman D, et al. Integrative molecular characterization of malignant pleural mesothelioma. Cancer Disco. 2018;8:1548-65.

7. Lee HS, Jang HJ, Choi JM, Zhang J, de Rosen VL, Wheeler TM, et al. Comprehensive immunoproteogenomic analyses of malignant pleural mesothelioma. JCI Insight. 2018;3::98575.

8. Lines JL, Sempere LF, Broughton T, Wang L, Noelle R. VISTA is a novel broad-spectrum negative checkpoint regulator for cancer immunotherapy. Cancer Immunol Res. 2014;2:510-7.

9. Nowak EC, Lines JL, Varn FS, Deng J, Sarde A, Mabaera R, et al. Immunoregulatory functions of VISTA. Immunol Rev. 2017; 276:66-79.
10. Le Mercier I, Chen W, Lines JL, Day M, Li J, Sergent P, et al. VISTA regulates the development of protective antitumor immunity. Cancer Res. 2014;74:1933-44.

11. Yan TD, Deraco M, Elias D, Glehen O, Levine EA, Moran BJ, et al. A novel tumor-node-metastasis (TNM) staging system of diffuse malignant peritoneal mesothelioma using outcome analysis of a multi-institutional database*. Cancer. 2011;117:1855-63.

12. Galateau-Salle F, Churg A, Roggli V, Travis WD. The 2015 World Health Organization Classification of Tumors of the Pleura: Advances since the 2004 Classification. J Thorac Oncol. 2016;11:142-54.

13. Maio M, Scherpereel A, Calabro L, Aerts J, Cedres Perez S, Bearz A, et al. Tremelimumab as second-line or third-line treatment in relapsed malignant mesothelioma (DETERMINE): a multicentre, international, randomised, double-blind, placebo-controlled phase 2b trial. Lancet Oncol. 2017;18:1261-73.

14. Lines JL, Pantazi E, Mak J, Sempere LF, Wang L, O’Connell S, et al. VISTA is an immune checkpoint molecule for human T cells. Cancer Res. 2014;74:1924-32.

15. Villarroel-Espindola F, Yu X, Datar I, Mani N, Sanmamed M, Velcheti V, et al. Spatially Resolved and Quantitative Analysis of VISTA/PD-1H as a Novel Immunotherapy Target in Human NonSmall Cell Lung Cancer. Clin Cancer Res. 2018;24:1562-73.

16. Mulati K, Hamanishi J, Matsumura N, Chamoto K, Mise N, Abiko K, et al. VISTA expressed in tumour cells regulates $\mathrm{T}$ cell function. Br J Cancer. 2019;120:115-27.

17. Husain AN, Colby TV, Ordonez NG, Allen TC, Attanoos RL, Beasley MB, et al. Guidelines for Pathologic Diagnosis of Malignant Mesothelioma 2017 Update of the Consensus Statement From the International Mesothelioma Interest Group. Arch Pathol Lab Med. 2018;142:89-108.

18. Hwang HC, Sheffield BS, Rodriguez S, Thompson K, Tse CH, Gown AM, et al. Utility of BAP1 immunohistochemistry and p16 (CDKN2A) FISH in the diagnosis of malignant mesothelioma in effusion cytology specimens. Am J Surg Pathol. 2016;40:120-6.

19. Hwang HC, Pyott S, Rodriguez S, Cindric A, Carr A, Michelsen $\mathrm{C}$, et al. BAP1 Immunohistochemistry and p16 FISH in the Diagnosis of Sarcomatous and Desmoplastic Mesotheliomas. Am J Surg Pathol. 2016;40:714-8.

20. Pasello G, Zago G, Lunardi F, Urso L, Kern I, Vlacic G, et al. Malignant pleural mesothelioma immune microenvironment and checkpoint expression: correlation with clinical-pathological features and intratumor heterogeneity over time. Ann Oncol. 2018;29:1258-65.

21. Awad MM, Jones RE, Liu H, Lizotte PH, Ivanova EV, Kulkarni $\mathrm{M}$, et al. Cytotoxic T Cells in PD-L1-positive malignant pleural mesotheliomas are counterbalanced by distinct immunosuppressive factors. Cancer Immunol Res. 2016;4:1038-48.

22. Ahn S, Choi IH, Han J, Kim J, Ahn MJ. Pleural mesothelioma: an institutional experience of 66 cases. Korean J Pathol. 2014;48:91-9. 